\title{
Engineering a thermostable Halothermothrix orenii $\beta$-glucosidase for improved galacto-oligosaccharide synthesis
}

\author{
Noor Hassan ${ }^{1} \cdot$ Barbara Geiger $^{2} \cdot$ Rosaria Gandini $^{1,4}$ - Bharat K. C. Patel $^{3}$. \\ Roman Kittl $^{2}$ - Dietmar Haltrich ${ }^{2}$ Thu-Ha Nguyen ${ }^{2}$. Christina Divne ${ }^{1,4}$. \\ Tien Chye Tan ${ }^{1,4}$
}

Received: 9 June 2015 /Revised: 19 October 2015 / Accepted: 24 October 2015 /Published online: 1 December 2015

(C) The Author(s) 2015. This article is published with open access at Springerlink.com

\begin{abstract}
Lactose is produced in large amounts as a byproduct from the dairy industry. This inexpensive disaccharide can be converted to more useful value-added products such as galacto-oligosaccharides (GOSs) by transgalactosylation reactions with retaining $\beta$-galactosidases (BGALs) being normally used for this purpose. Hydrolysis is always competing with the transglycosylation reaction, and hence, the yields of GOSs can be too low for industrial use. We have reported that a $\beta$ glucosidase from Halothermothrix orenii (HoBGLA) shows promising characteristics for lactose conversion and GOS synthesis. Here, we engineered HoBGLA to investigate the possibility to further improve lactose conversion and GOS production. Five variants that targeted the glycone $(-1)$ and aglycone (+1) subsites (N222F, N294T, F417S, F417Y, and Y296F) were designed and expressed. All variants show significantly impaired catalytic activity with cellobiose and lactose as substrates. Particularly, F417S is hydrolytically crippled with cellobiose as substrate with a 1000-fold decrease
\end{abstract}

Noor Hassan and Barbara Geiger were joint first authors.

Tien Chye Tan

tantc@kth.se

1 AlbaNova University Center, School of Biotechnology, KTH Royal Institute of Technology, Roslagstullsbacken 21,

S-10691 Stockholm, Sweden

2 Food Biotechnology Laboratory, BOKU-University of Natural Resources and Life Sciences Vienna, 1190 Vienna, Austria

3 Microbial Gene Research and Resources Facility, School of Biomolecular and Physical Sciences, Griffith University, Brisbane QLD 4111, Australia

4 Department of Medical Biochemistry and Biophysics, Karolinska Institute, Scheelelaboratoriet, Scheeles väg 2,

S-17177 Stockholm, Sweden in apparent $k_{\text {cat }}$, but to a lesser extent affected when catalyzing hydrolysis of lactose ( 47 -fold lower $k_{\text {cat }}$ ). This large selective effect on cellobiose hydrolysis is manifested as a change in substrate selectivity from cellobiose to lactose. The least affected variant is $\mathrm{F} 417 \mathrm{Y}$, which retains the capacity to hydrolyze both cellobiose and lactose with the same relative substrate selectivity as the wild type, but with $\sim 10$-fold lower turnover numbers. Thin-layer chromatography results show that this effect is accompanied by synthesis of a particular GOS product in higher yields by Y296F and F417S compared with the other variants, whereas the variant $\mathrm{F} 417 \mathrm{Y}$ produces a higher yield of total GOSs.

Keywords $\beta$-Glucosidase $\cdot \beta$-Galactosidase $\cdot$

Halothermophile $\cdot$ Halothermothrix $\cdot$ Lactose conversion .

Galacto-oligosaccharides · Transglycosylation mutants

\section{Introduction}

About 150-200 million tons of lactose are generated each year from liquid whey (Smithers, 2008). Lactose can be conveniently hydrolyzed into glucose and galactose by the use of $\beta$-galactosidases (BGALs). In cases where the enzyme displays significant transglycosylation activity, hydrolysis can be combined with the transfer of hydrolysis products onto suitable acceptor molecules to form new value-added compounds such as galacto-oligosaccharides (GOSs).

The main producers of GOSs are BGALs produced by various microorganisms such as Aspergillus oryzae, Aspergillus niger, Kluyveromyces lactis, and Kluyveromyces fragilis (Torres et al. 2010; Gosling et al. 2010). A number of commercial and non-commercial BGALs from different sources have been evaluated for their ability and efficiency 
to produce GOSs (e.g., Petzelbauer et al. 2000; Jørgensen et al. 2001; Hung et al. 2002; Chockchaisawasdee et al. 2005; Nguyen et al. 2006; Nakkharat \& Haltrich, 2006; Splechtna et al. 2007; Goulas et al. 2009; Neri et al. 2009; Maischberger et al. 2010; Iqbal et al. 2011; Liu et al. 2011; Rodriguez-Colinas et al. 2011; 2012; 2013; Nguyen et al. 2012; Osman et al. 2012; Urrutia et al. 2013; Wu et al. 2013; Yu \& Sullivan, 2013; Arreola et al. 2014). A. oryzae, Bacillus circulans, Cryptococcus laurentii, K. lactis, and Streptococcus thermophilus are commercial sources of BGALs used for GOS production. When using these enzymes, yields of GOS formation ranging from 14 to $45 \%$ are reported with $5 \%$ initial lactose concentrations (Torres et al. 2010; Gosling et al. 2010). The use of rational design and enzyme engineering offers a means by which to improve the transglycosylation-to-hydrolysis $(\mathrm{T} / \mathrm{H})$ ratio to produce more useful enzyme variants giving higher GOS yields.

Alternatives to BGALs for these applications include many retaining $\beta$-glucosidases (BGL) that are capable of catalyzing transglycosylation as a side reaction to the functionally relevant hydrolytic cleavage of glycosidic bonds. Efforts to engineer BGLs for improved transglycosylation have been reported in the literature (Hansson et al. 2001; Feng et al. 2005; $\mathrm{Wu}$ et al. 2013), where the typical engineering strategy involves replacement of side chains in the aglycone $(+1)$ and glycone (-1) subsites (Hansson et al. 2001; Feng et al. 2005). For engineering, it is advantageous to start with an enzyme that has evolved naturally to be functional under conditions that are relevant for the desired application, for instance high temperature. Thermophilic and hyperthermophilic bacteria are among the most useful microbial producers of highly stable and robust enzymes for bioprocesses. Among others, Halothermothrix orenii is a heterotrophic, halophilic, thermophilic, obligate anaerobic bacterium (Cayol et al. 1994) that produces a thermostable $\beta$-glucosidase, $H o B G L A$ (Mijts \& Patel, 2001; Mavromatis et al. 2009; Kori et al. 2011; Hassan et al. 2015). We have reported previously the biochemical and structural characterization of HoBGLA and shown that this GH1 glycosidase (www.cazy.org; Lombard et al. 2014) displays attractive properties relevant to GOS synthesis (Hassan et al. 2015). Specifically, the enzyme has $\beta$-galactosidase activity at high temperatures $\left(65-70^{\circ} \mathrm{C}\right)$ and within a broad $\mathrm{pH}$ range (4.5-7.5), conditions under which the wild-type enzyme also displays significant transgalac tosylation activity to efficiently convert lactose into mainly $\beta$-D-Galp-( $1 \rightarrow 3)$-D-Lac (3'-galactosyl lactose; 3GALA) and $\beta$-D-Galp-( $1 \rightarrow 6)$-D-Lac (6'-galactosyl lactose; 6GALA).

Encouraged by the high natural transgalactosylation activity of HoBGLA, and the general amenability of BGLs to be engineered toward improved $\mathrm{T} / \mathrm{H}$ ratios, we set out in this study to investigate the possibility to further improve transgalactosylation activity and GOS yield for HoBGLA by replacing amino acid side chains in, or near, the -1 subsite in positions previously shown to improve transglycosylation for related BGLs (Hansson et al. 2001; Feng et al. 2005; Lundemo et al. 2013; Teze et al. 2014). We present the production, biochemical characterization, and transgalactosylation analysis for six HoBGLA active-site variants aimed at improving GOS production.

\section{Materials and methods}

\section{Site-directed mutagenesis, expression, and purification of HoBGLA variants}

The cloning and expression of the H. orenii bglA gene (UniProtKB B8CYA8) have been reported previously (Hassan et al. 2015). The wild-type bglA gene cloned in the pNIC28-Bsa4 vector containing a cleavable N-terminal hexahistidine tag and the tobacco etch virus (TEV) protease cleavage site (sequence ${ }^{23}$ MHHHHHHSSGVDLGTEN LYFQSM $^{-1}$ ) (Savitsky et al. 2010) was used as template for site-directed mutagenesis to produce the single-replacement variants N222F, N294T, Y296F, N406I, F417Y, and F417S. Forward and reverse PCR primers were designed with the QuickChange ${ }^{\circledR}$ Primer Design Program from Agilent Technology. The forward primers are given in Table 1, and the reverse primers were the reverse complements of the forward primers. All PCR reactions, plasmid transformations, expression, cell harvest, and protein purification were performed as described for the HoBGLA wild-type and active-site variants reported earlier (Hassan et al. 2015). Briefly, Escherichia coli BL21(DE3) cells were grown

Table 1 Forward PCR mutagenesis primers

\begin{tabular}{ll}
\hline N222F_fwd & 5'-CGGGTAAGCAGGGGTTAAGAAGAGAGTAATACCAATCTCC-3' $^{\prime}$ \\
\hline N294T_fwd & 5'-CCATCCTGGAGTAGTAAGTAATGCCCAGGAAGTCAA-3' \\
Y296F_fwd & 5'-ACCACCATCCTGGAGAAGTAATTAATGCCCAGGAA-3' \\
N406I_fwd & 5'-GCCATAGGCCCATTCAAAAATATCCATCAATGACCACAC-3' \\
F417Y_fwd & 5'-CCTATGGCTATAGCAAGCGCTATGGTCTCATTTATG-3' \\
F417S_fwd & 5'-TAATCAACATAAATGAGACCACTGCGCTTGCTATAGCCATAGGC-3' \\
\hline
\end{tabular}


in Terrific Broth (TB) medium supplemented with $50 \mu \mathrm{g} / \mathrm{mL}$ kanamycin and glycerol as carbon source $(60 \mathrm{~mL}$ per $600 \mathrm{~mL}$ ), induced with $0.2 \mathrm{mM}$ IPTG and cultivated at $18{ }^{\circ} \mathrm{C}$ for $16-18 \mathrm{~h}$. Purification was performed as described previously (Hassan et al. 2015), using an initial step of $\mathrm{Ni}^{2+}$ charged immobilized metal affinity chromatography (IMAC), after which the $\mathrm{His}_{6}$ tag was removed using tobacco etch virus (TEV) protease. After tag removal, a second step of IMAC was performed (i.e., reverse IMAC) where the TEV-treated target protein lacking the $\mathrm{His}_{6}$ tag was collected in the flow through and further purified using size exclusion chromatography on a HiLoad ${ }^{\text {TM }} 16 / 60$ Superdex $^{\text {TM }} 200$ prep grade column (GE Healthcare Life Sciences) equilibrated with $20 \mathrm{mM}$ HEPES (pH 7.0) and $150 \mathrm{mM} \mathrm{NaCl}$.

\section{Hydrolytic activity assays of the HoBGLA variants on cellobiose and lactose}

The hydrolytic activity of $H o$ BGLA variants on cellobiose and lactose was assayed using the coupled glucose oxidase/ peroxidase assay (Kunst et al. 1988) as described earlier for wild-type and mutant $H o$ BGLA (Hassan et al. 2015), the only differences being the buffer and temperature at which the assay was performed. The reactions were carried out at $70{ }^{\circ} \mathrm{C}$ in $20 \mathrm{mM}$ HEPES buffer (pH 7.0) and $0.15 \mathrm{M} \mathrm{NaCl}$ with substrate concentrations in the range 2 to $120 \mathrm{mM}$ for cellobiose, and 5 to $400 \mathrm{mM}$ for lactose. Enzyme concentrations used were as follows: wild type, $0.085 \mathrm{mg} / \mathrm{mL}$; N222F, $1.7 \mathrm{mg} /$ $\mathrm{mL}$; N294T, $4 \mathrm{mg} / \mathrm{mL}$; Y296F, $5 \mathrm{mg} / \mathrm{mL}$; F417S, $8.5 \mathrm{mg} /$ $\mathrm{mL}$; and F417Y, $4 \mathrm{mg} / \mathrm{mL}$. One unit of lactose-hydrolyzing activity was defined as the amount of enzyme releasing $1 \mu \mathrm{mol}$ of D-glucose per minute under the given conditions. One unit of cellobiose-hydrolyzing activity was defined as the amount of enzyme releasing $2 \mu \mathrm{mol}$ of D-glucose per minute under similar conditions as described for determination of $\beta$-galactosidase activity using lactose as the substrate. Non-linear regression was used to derive the kinetic parameters, and the data were fitted to the Michaelis-Menten model using GraphPad Prism 6.0 for Mac (GraphPad Software, San Diego, CA, USA, www.graphpad.com). The apparent turnover values $\left(k_{\text {cat,app }}\right)$ were calculated using the experimentally determined $v_{\max }$ values and a molecular mass of $53 \mathrm{kDa}$ for the enzyme.

\section{TLC screening for GOS production by HoBGLA variants using cell lysates}

To assess the transglycosylation activity of the HoBGLA variants, synthesized GOS products were screened by thin-layer chromatography (TLC). To this end, the crude-cell extracts were incubated in the presence of $30 \%$ lactose $(w / v)$ at $70{ }^{\circ} \mathrm{C}$ and different durations, allowing denaturation of most endogenous $E$. coli proteins. Specifically, 2-mL overnight cultures of $E$. coli BL21(DE3) cells carrying the $p$ NIC28Bsa4-HoBGLA vectors were harvested by centrifugation, and the cell pellet resuspended in either $200 \mu \mathrm{L}$ sodium phosphate buffer, pH 6.0, containing $300 \mathrm{~g} / \mathrm{L}$ lactose, and $1 \mathrm{mM} \mathrm{Mg}^{2+}$ to increase enzyme stability (Nguyen et al. 2006; Iqbal et al. 2011; Hassan et al. 2015), or $200 \mu \mathrm{L}$ sodium phosphate buffer, pH 6.0, containing $200 \mathrm{~g} / \mathrm{L}$ cellobiose and $1 \mathrm{mM} \mathrm{Mg}^{2+}$. Cells were lysed by ultrasonication on ice. The resulting cell lysates were incubated at $70{ }^{\circ} \mathrm{C}$ with shaking $(700$ r.p.m.). In the case of lactose as substrate, the reaction was run for 3 and $4.5 \mathrm{~h}$, after which samples were taken for analysis of GOS products. The samples were heated at $95^{\circ} \mathrm{C}$ for $5 \mathrm{~min}$ and diluted $1: 10$, followed by loading of $1 \mu \mathrm{L}$ sample on a TLC plate. In the case of cellobiose as substrate, the reaction was carried out for $2 \mathrm{~h}$ after which the enzymes were heat-inactivated for $5 \mathrm{~min}$ at $95{ }^{\circ} \mathrm{C}$, and the carbohydrate content analyzed using TLC ( $2 \mu \mathrm{L}$ of 1:10 dilution). HPTLC Li Chrosper ${ }^{\circledR}$ Silica gel 60 $\mathrm{F}_{254} \mathrm{~S}$ (Merck) was used as adsorbent. Samples were applied on the plates and placed in the eluent ( $n$-butanol- $n$-propanolethanol-water $=2: 3: 3: 2$ ). Visualization of the separated carbohydrates was performed by immersing the TLC plate in a staining solution $(0.5 \mathrm{~g}$ thymol, $95 \mathrm{~mL} 96 \%$ ethanol, $5 \mathrm{~mL}$ concentrated sulfuric acid) for $3 \mathrm{~s}$ and subsequent heating at $90{ }^{\circ} \mathrm{C}$ for approximately $1 \mathrm{~min}$. Standards for lactose conversion included a mixture of glucose, galactose, and lactose (LGG); a purified GOS mixture with monosaccharides and lactose removed produced using Lactobacillus sp. $\beta$-galactosidase (Maischberger et al. 2008); and Vivinal ${ }^{\circledR}$ GOS (Borculo Domo, NL). For cellobiose conversion, cellobiose and glucose were used as standards.

For HPLC analysis of transglycosylation products obtained with purified enzymes, a volume of $50 \mu \mathrm{L}$ enzyme $(7 \mathrm{mg} / \mathrm{mL})$ was mixed with $450 \mu \mathrm{L}$ lactose $(300 \mathrm{~g} / \mathrm{L})$ in $50 \mathrm{mM}$ sodium phosphate buffer ( $\mathrm{pH} \mathrm{6}$ ) containing $1 \mathrm{mM} \mathrm{MgCl} 2$ and incubated with shaking at $70{ }^{\circ} \mathrm{C}$ at 600 r.p.m. for $12 \mathrm{~h}$. Samples were withdrawn at different time points and heat-inactivated for $5 \mathrm{~min}$ at $95^{\circ} \mathrm{C}$, followed by HPLC analysis as described previously (Spechtna et al. 2007).

\section{Transgalactosylation of lactose using purified enzymes and analysis of galacto-oligosaccharides}

A volume of $50 \mu \mathrm{L}$ purified enzyme $(7 \mathrm{mg} / \mathrm{mL})$ of $H o B G L A$ variants was mixed with $450 \mu \mathrm{L}$ lactose $(300 \mathrm{~g} / \mathrm{L})$ in $50 \mathrm{mM}$ sodium phosphate buffer ( $\mathrm{pH}$ 6) containing $1 \mathrm{mM} \mathrm{MgCl}$ and incubated with shaking at $70{ }^{\circ} \mathrm{C}$ at 600 r.p.m. for $12 \mathrm{~h}$. Samples were withdrawn at specific time intervals and immediately transferred to $99^{\circ} \mathrm{C}$ for $5 \mathrm{~min}$ to inactivate the enzyme. Samples were stored at $-18{ }^{\circ} \mathrm{C}$ for subsequent analysis.

The GOS mixtures were analyzed by high-performance anion exchange chromatography with pulsed amperometric detection (HPAEC-PAD). HPAEC-PAD analysis was carried 
out on a Dionex DX-500 system consisting of a GP50 gradient pump, an ED 40 electrochemical detector with a gold working electrode and an $\mathrm{Ag} / \mathrm{AgCl}$ reference electrode, and Chromeleon version 6.5 (Dionex Corp., Sunnyvale, CA). All eluents were degassed by flushing with helium for $30 \mathrm{~min}$. Separations were performed at room temperature on a CarboPac PA-1 column $(4 \mathrm{~mm} \times 250 \mathrm{~mm})$ connected to a CarboPac PA-1 guard column (Dionex). Separation of D-glucose-, D-galactose, lactose, and allolactose was carried out with an isocratic run (45 min) with $15 \mathrm{mM} \mathrm{NaOH}$ at $1.0 \mathrm{~mL} / \mathrm{min}$, followed by 25 -min elution with $100 \mathrm{mM} \mathrm{NaOH}$ (gradient 1). For separation of other GOSs, eluents A $(100 \mathrm{mM} \mathrm{NaOH})$ and $\mathrm{B}(100 \mathrm{mM} \mathrm{NaOH}$ and $150 \mathrm{mM} \mathrm{NaAc})$ were mixed to form the following gradient: $98 \% \mathrm{~A}$ from 0 to $10 \mathrm{~min}, 98 \% \mathrm{~A}$ to $52 \% \mathrm{~A}$ from 10 to $40 \mathrm{~min}$, and then $52 \% \mathrm{~A}$ for another $5 \mathrm{~min}$ (gradient 2). The column was washed with $20 \% \mathrm{~B}$ for $10 \mathrm{~min}$ and re-equilibrated for $15 \mathrm{~min}$ with the starting conditions of the employed gradient.

Individual GOS components were identified by comparison to authentic material, specifically $\beta$-D-Galp- $(1 \rightarrow 3)-\mathrm{D}-\mathrm{G}$ lc, $\beta$-D-Gal $p$ - $(1 \rightarrow 6)$-D-Glc, $\beta$-D-Gal $p$-( $1 \rightarrow 3)$-D-Gal, $\beta$-DGal $p$ - $(1 \rightarrow 4)$-D-Gal, $\beta$-D-Galp-( $\rightarrow$ 6)-D-Gal, $\beta$-DGalp-(1 $\rightarrow$ 3)-D-Lac, $\beta$-D-Galp-( $1 \rightarrow 4)$-D-Lac, and $\beta$-DGalp-(1 $\rightarrow 6)$-D-Lac purchased from Carbosynth (Berkshire, UK). The degree of lactose conversion was calculated as percentage of lactose converted of initial lactose employed. The GOS yields were calculated as percentage of GOSs formed of total sugars.

\section{Results}

\section{Construction and expression of HoBGLA variants}

Based on engineering studies on related GH1 $\beta$-glycosidases (Hansson et al. 2001; Feng et al. 2005; Wu et al. 2013), amino acids in the glycone (-1) and aglycone (+1) subsites of $H o$ BGLA were selected for mutagenesis with the aim to enhance the T/H ratio. The $\beta$-glucosidase $T n B g l 1 \mathrm{~A}$ from Thermotoga neapolitana was engineered toward improved transglycosylation by replacing $\mathrm{N}^{220}$ in subsite +1 with phenylalanine. In $T n$ Bgll A, this substitution caused significant improvement of the $\mathrm{T} / \mathrm{H}$ ratio and higher yields of alkyl glycosides through transglycosylation (Lundemo et al. 2013). The corresponding residue in HoBGLA is $\mathrm{N}^{222}$, and thus, the $H o B G L A$ variant $\mathrm{N} 222 \mathrm{~F}$ was considered an interesting candidate. Introducing a phenylalanine side chain would increase hydrophobicity of the +1 subsite and possibly increase the affinity for acceptors of less polar character than water, such as sugars, which theoretically may favor a higher $\mathrm{T} / \mathrm{H}$ ratio.

In the case of Thermus thermophilus $\beta$-glycosidase $\mathrm{Tt} \beta$ gly, directed-evolution experiments identified the replacements of N282T, N390I, and F401S in the vicinity of the -1 subsite as variants with improved $\mathrm{T} / \mathrm{H}$ ratios (Feng et al. 2005; Teze et al. 2014). These residues are conserved in HoBGLA and correspond to $\mathrm{N}^{294}, \mathrm{~N}^{406}$, and $\mathrm{F}^{417}$. Moreover, the transgalactosylation activity increased by $22 \%$ for the Pyrococcus furiosus $\beta$-glucosidase CelB variant $\mathrm{F} 426 \mathrm{Y}$ ( $\mathrm{F}^{417}$ in HoBGLA) at low lactose concentrations compared with the wild type (Hansson et al. 2001). A comparison of the crystal structures of $\mathrm{Tt} \beta$-gly N282T (PDB code 4BCE; Teze et al. 2014) and F401S (PDB code 3ZJK; Teze et al. 2014) with that of HoBGLA (PDB code 4PTX; Hassan et al. 2015) suggested that these replacements may cause similar effects in HoBGLA. Based on the above information, the HoBGLA variants N294T, N406I, F417S, and F417Y were selected. Additional $\mathrm{Tt} \beta$-gly variants with promising transglycosylation characteristics have been reported, such as R75A, W120C, N163A, and Y284F (Teze et al. 2014). These positions correspond to $\mathrm{R}^{77}, \mathrm{~W}^{122}, \mathrm{~N}^{165}$, and $\mathrm{Y}^{296}$ in HoBGLA, respectively, and of these, the HoBGLA Y296F replacement in subsite -1 was considered for further work.

In total, six $H o B G L A$ variants were designed rationally, including N222F, N294T, Y296F, N406I, F417Y, and F417S (Fig. 1), of which all but N406I could be expressed. The expression yields for the purified proteins (after reverse IMAC) were as follows: wild type, $7.5 \mathrm{mg} / \mathrm{L}$ culture $(0.8 \mathrm{mg} / \mathrm{g}$ of wet cell mass $) ; \mathrm{N} 222 \mathrm{~F}, 1.0 \mathrm{mg} / \mathrm{L}$ culture $(0.14 \mathrm{mg} / \mathrm{g}$ of wet cell mass); N294T, $2.9 \mathrm{mg} / \mathrm{L}$ culture $(0.5 \mathrm{mg} / \mathrm{g}$ of wet cell mass); N406I, no expression; F417S, $3.8 \mathrm{mg} / \mathrm{L}$ culture $(0.9 \mathrm{mg} / \mathrm{g}$ of wet cell mass $) ; \mathrm{F} 417 \mathrm{Y}, 2.5 \mathrm{mg} /$ L culture $(0.4 \mathrm{mg} / \mathrm{g}$ of wet cell mass); and Y296F, $3.2 \mathrm{mg} / \mathrm{L}$ culture $(0.5 \mathrm{mg} / \mathrm{g}$ of wet cell mass).

\section{Characterization of the hydrolytic activity of HoBGLA mutants}

The kinetic parameters $\left(v_{\max }, K_{\mathrm{m}}, k_{\mathrm{cat}} / K_{\mathrm{m}}\right)$ for hydrolysis of cellobiose and lactose were determined for the HoBGLA wild type and variants (Table 2). We previously reported the kinetics for the HoBGLA-catalyzed hydrolysis of cellobiose and lactose at $50^{\circ} \mathrm{C}$ (Hassan et al. 2015). The turnover number for cellobiose hydrolysis catalyzed by the wild type increases slightly $(\sim 6 \%)$ when raising the temperature to $70{ }^{\circ} \mathrm{C}$, while a larger increase in apparent $k_{\text {cat }}$ is observed for lactose hydrolysis ( $\sim 0 \% \%)$. The changes in the $K_{\mathrm{m}}$ values in response to increasing temperature are more pronounced with $K_{\mathrm{m} \text { [cellobiose] }}$ decreasing 2.5 -fold and $K_{\text {m[lactose] }}$ dropping 6-fold. These changes are manifested as a 2.7 -fold and 9-fold increase in specificity constant for cellobiose and lactose, respectively. This shows that wild-type HoBGLA achieves improved specificity and catalytic efficiency for both cellobiose and lactose at $70{ }^{\circ} \mathrm{C}$. Compared with the wild-type enzyme, all expressed variants show impaired hydrolytic activity for both substrates. 
Fig. 1 Structural details of mutation sites. a The active site of wild-type $H o B G L A$ with relevant residues shown (PDB code 4PTX; Hassan et al. 2015). The amino acid replacements were modeled in the crystal structure: b N222F, c N294T, d Y296F, e N406I, f F417Y, and g F417S. Mutated residues are highlighted in green. A previously modeled 3GALA molecule (Hassan et al. 2015) was shown to delineate the subsites $-1,+1$, and +2 and has been superimposed on the theoretical structural models of the variants (Color figure online)
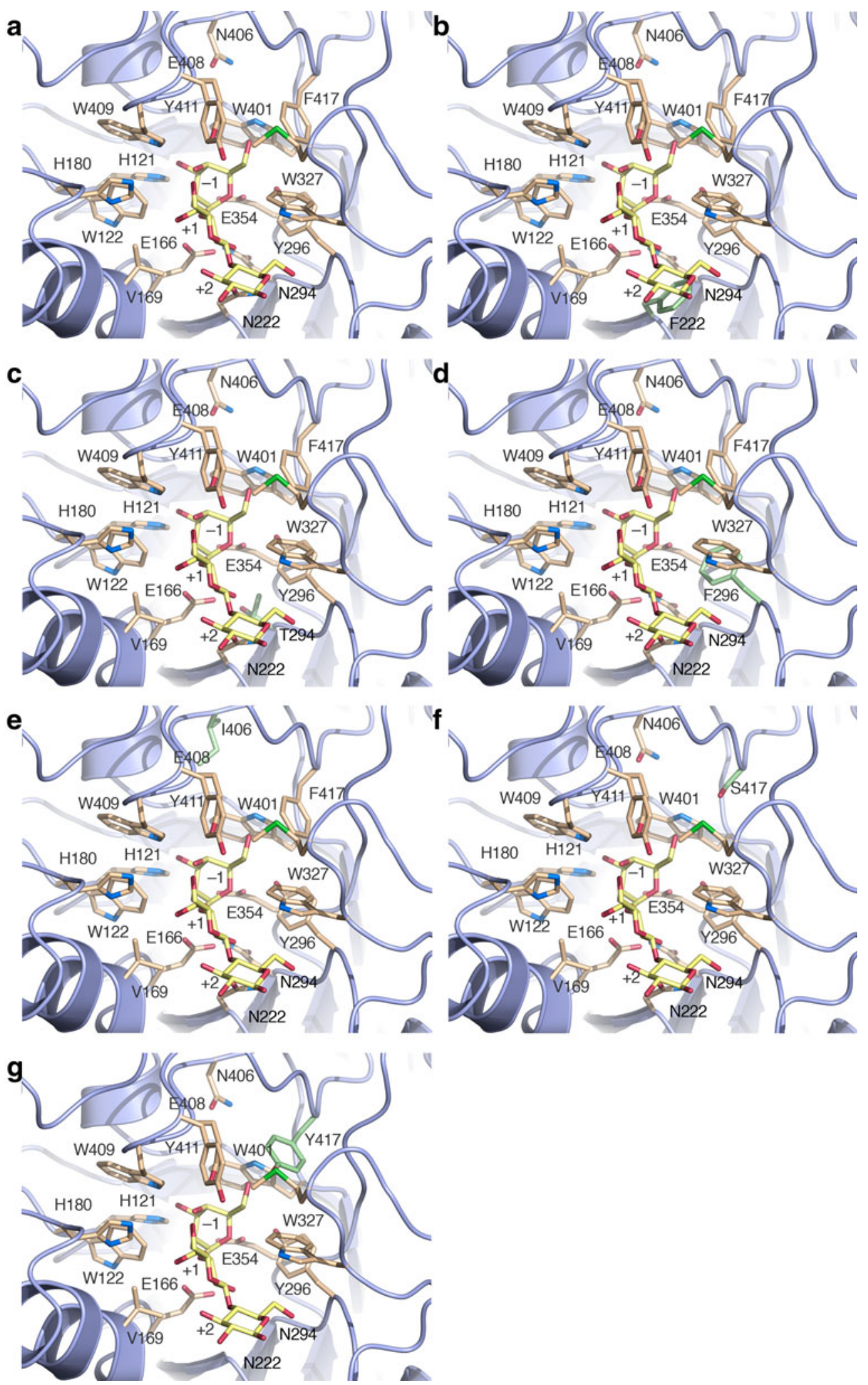

The variant $\mathrm{N} 222 \mathrm{~F}$ shows an $\sim 23$-fold decrease in $k_{\mathrm{cat}}$, and 9 -fold increase in $K_{\mathrm{m}}$ for cellobiose hydrolysis. The variant is also severely compromised catalytically when lactose is used as substrate ( $\sim 250$-fold lower $k_{\text {cat }}$ and $\sim 1$.6-fold increase in $\left.K_{\mathrm{m}}\right)$. For cellobiose hydrolysis, the variant N294T shows an $\sim 23$-fold decrease in $k_{\text {cat }}$ (as for $\mathrm{N} 222 \mathrm{~F}$ ), while $K_{\mathrm{m}}$ remains 
Table 2 Kinetic parameters for $\beta$-glucosidase and $\beta$-galactosidase activities

\begin{tabular}{lccccccc}
\hline & D-cellobiose & \multicolumn{3}{c}{ D-lactose } & Selectivity, cellobiose over lactose $^{\text {b }}$ \\
\hline & $k_{\text {cat,app }}$ & $K_{\mathrm{m}}$ & $k_{\text {cat,app }} / K_{\mathrm{m}}$ & $k_{\text {cat,app }}$ & $K_{\mathrm{m}}$ & $k_{\text {cat,app }} / K_{\mathrm{m}}$ & $\left(k_{\text {cat,app }} / K_{\mathrm{m}[\mathrm{cel}]}\right) /$ \\
$(\mathrm{mM})$ & $\left(\mathrm{mM}^{-1} \mathrm{~s}^{-1}\right)$ & $\left(\mathrm{s}^{-1}\right)$ & $(\mathrm{mM})$ & $\left(\mathrm{s}^{-1} \mathrm{mM}^{-1}\right)$ & 1.54 & 9.4 \\
$\mathrm{WT}^{\mathrm{a}}$ & 366 & 25.4 & 14.4 & 231 & 154 & 1.9 & 2.8 \\
$\mathrm{WT}$ & $387 \pm 15$ & $10.0 \pm 1.0$ & 38.7 & $366 \pm 17$ & $26.3 \pm 2.8$ & 13.9 & 5.0 \\
$\mathrm{~N} 222 \mathrm{~F}$ & $16.9 \pm 1.3$ & $93.2 \pm 12.6$ & 0.18 & $1.47 \pm 0.05$ & $41.3 \pm 3.1$ & 0.036 & 7.5 \\
$\mathrm{~N} 294 \mathrm{~T}$ & $16.5 \pm 0.4$ & $9.0 \pm 0.8$ & 1.8 & $7.4 \pm 0.2$ & $30.8 \pm 2.0$ & 0.24 & 1.8 \\
Y296F & $8.5 \pm 0.3$ & $20.2 \pm 1.6$ & 0.42 & $6.9 \pm 0.2$ & $29.1 \pm 2.3$ & 0.24 & 2.8 \\
F417Y & $38.7 \pm 0.6$ & $3.5 \pm 0.2$ & 11.1 & $41.5 \pm 1.2$ & $10.3 \pm 0.9$ & 4.0 & 0.09 \\
F417S & $0.38 \pm 0.01$ & $11.9 \pm 0.9$ & 0.032 & $7.8 \pm 0.4$ & $23.2 \pm 2.7$ & 0.34 & \\
\hline
\end{tabular}

${ }^{\text {a }}$ Reaction performed at $50{ }^{\circ} \mathrm{C}$; from Hassan et al. 2015 ; all other reactions performed at $70{ }^{\circ} \mathrm{C}$

${ }^{\mathrm{b}}$ A ratio $>1$ favors cellobiose hydrolysis, whereas a ratio $<1$ favors lactose hydrolysis

unchanged compared with the wild type. Using lactose as substrate, this mutant displays an almost 50 -fold decrease in $k_{\text {cat }}$ and only slightly higher $K_{\mathrm{m}}(17 \%)$. The HoBGLA variant Y296F hydrolyzes cellobiose $\sim 45$ times slower than does the wild-type enzyme, and the affinity for cellobiose is reduced, as indicated by a twofold increase in $K_{\mathrm{m}}$ value. Turnover of lactose is affected slightly more ( $\sim 53$-fold lower $\left.k_{\text {cat }}\right)$, but with only minor impact on $K_{\mathrm{m}}$ (11\% increase).

The least affected $H o B G L A$ variant is F417Y, which, compared to the wild type, retains 10 and $11 \%$ of the cellobiose and lactose turnover numbers, respectively. A concomitant drop in $K_{\mathrm{m}}$ and 3.5-fold decrease in specificity constant for both substrates accompany the decrease in apparent $k_{\text {cat }}$. With cellobiose as substrate, the variant F417S shows the lowest turnover number $\left(k_{\text {cat }} 0.38 \mathrm{~s}^{-1}\right)$, which corresponds to a 1000 -fold decrease in $k_{\text {cat }}$, but an unperturbed $K_{\mathrm{m}}$ value. However, hydrolysis of lactose by this variant is less affected with a 47 -fold decrease in turnover number $\left(k_{\text {cat }}\right.$ $7.8 \mathrm{~s}^{-1}$ ) and similar $K_{\mathrm{m}}$ compared with the wild type. Thus, the F417S replacement in HoBGLA is detrimental for cellobiose hydrolysis whereas this variant remains relatively competent with respect to lactose hydrolysis.

\section{TLC screening for GOS production by HoBGLA variants using cell lysates}

We have reported previously that wild-type $H o$ BGLA is able to transform lactose into GOSs efficiently and in high yields (Hassan et al. 2015). As an initial evaluation of the capacity of the HoBGLA variants to synthesize GOSs, the crude lysates containing the respective variants were screened for their ability to hydrolyze cellobiose, and to convert lactose to products other than the hydrolysis products glucose and galactose using TLC (Fig. 2). As expected from the steady state kinetics (Table 2), all variants show hydrolytic activity on both cellobiose and lactose. The product patterns for cellobiose hydrolysis are similar for wild type, N222F, N294T, and F417Y (Fig. 2a). The absence of hydrolysis products from cellobiose for Y296F and F417S (Fig. 2a) under the conditions considered and the low associated turnover numbers, especially for F417S (Table 2), are consistent with poor performance of these mutants with cellobiose as substrate.

The variant $\mathrm{F} 417 \mathrm{Y}$ displays product patterns from lactose hydrolysis similar to those of the wild type, whereas N222F, N294F, Y296F, and F417S show different patterns of GOSs formed (Fig. 2b, c). The variants Y296F, F417Y, and F417S seem to generate especially one GOS product in higher yields (i.e., the lower GOS band in Fig. 2b, c). Of these, F417Y shows the weakest signal for residual lactose, which reflects the higher $k_{\text {cat,lac }}$ value of this variant compared with Y296F and F417S. As mentioned above, F417S shows a change in substrate selectivity from cellobiose to lactose, which could be useful for GOS production from substrate sources containing a mixture of cellobiose and lactose.

\section{Analysis of transgalactosylation activity and GOS production by HoBGLA variants}

The transgalactosylation activity of the HoBGLA variants was subsequently investigated in more detail using purified enzyme preparations. The reactions were performed at $70{ }^{\circ} \mathrm{C}$ with an initial lactose concentration of $300 \mathrm{~g} / \mathrm{L}$ using the same amount of purified enzyme $(\sim 0.35 \mathrm{mg})$. Three variants, Y296F, F417S, and F417Y, show improved total GOS yields compared with the wild-type enzyme (Table 3). The highest GOS yield of $\sim 57 \%$ was obtained with F417Y, and F417Y also shows highest lactose conversion after $8 \mathrm{~h}$ among the variants tested. N294T is the least efficient in converting lactose and forming GOSs compared with the other variants and the wild-type enzyme under the conditions applied here. 
a
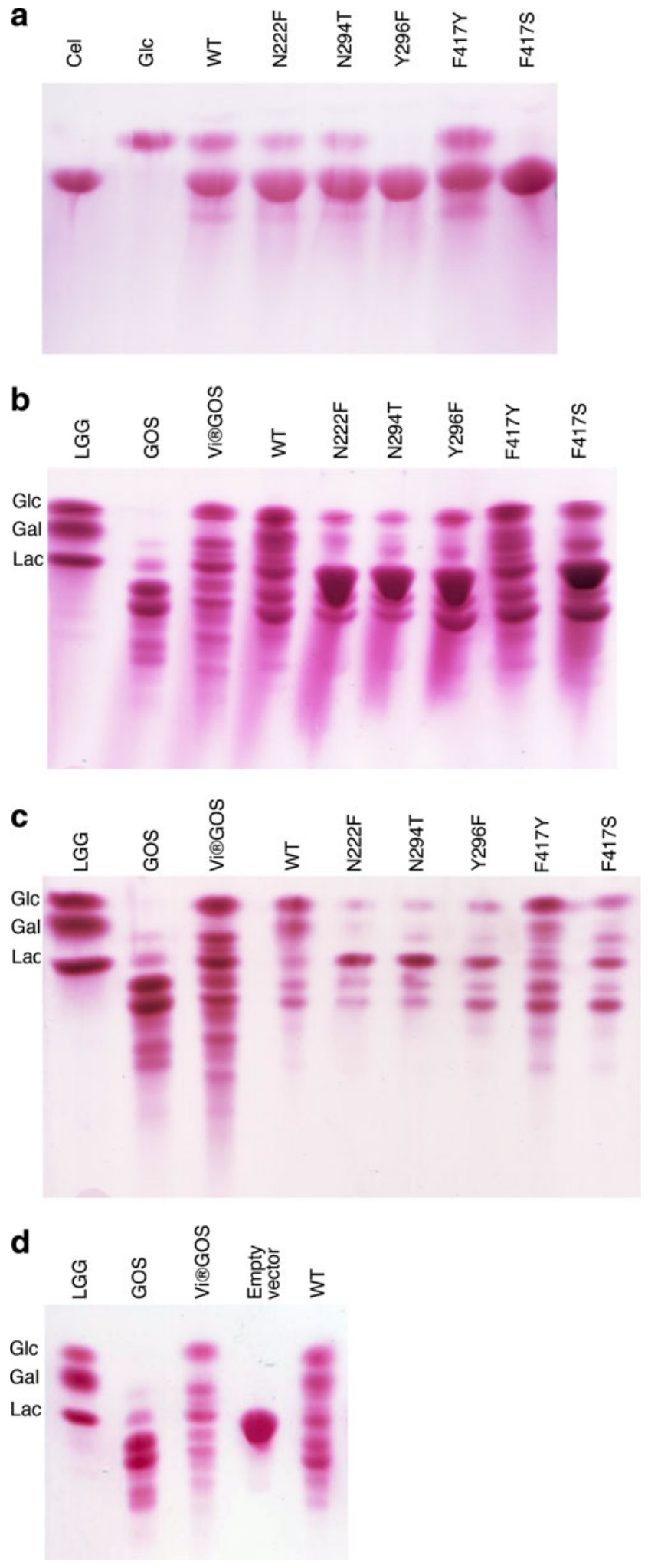

All variants yield $\beta$-D-Gal $p$ - $(1 \rightarrow 6)$-Lac as the predominant oligosaccharide product (Table 3), at levels ranging from 43 to $67 \%$ mass of the total GOSs produced. Y296F and F417S show almost identical GOS yields as well as the
Fig. 2 Cellobiose and lactose hydrolysis and transglycosylation using cell lysates. TLC analysis of HoBGLA-catalyzed hydrolysis and transglycosylation in cell lysates of a cellobiose after $2 \mathrm{~h}, \mathbf{b}$ lactose after $3 \mathrm{~h}$, c lactose after $4.5 \mathrm{~h}$; and $\mathbf{d}$ lactose after $4.5 \mathrm{~h}$ of reaction using cells carrying the expression vector without HoBGLA insert as control. Standards used: LGG, lactose (Lac); galactose (Gal); glucose (Glc); GOS, purified GOS with monosaccharides and lactose removed; Vivinal ${ }^{\mathbb{R}}$ GOS; Cel, cellobiose

highest relative amounts of $\beta$-D-Gal $p-(1 \rightarrow 6)$-Lac. Differences between these two variants during lactose transformation are that F417S converts lactose faster and that F417S forms the disaccharide $\beta$-D-Galp-( $1 \rightarrow 3)$-Glc as its second most frequent GOS product, whereas this is $\beta$-D-Galp- $(1 \rightarrow$ 3 )-Lac in the case of Y296F. Only four main GOS components were found in detectable amounts in the GOS mixtures formed when using Y296F and F417S, which were $\beta$-DGal $p$ - $(1 \rightarrow 6)$-Lac, $\beta$-D-Gal $p$ - $(1 \rightarrow 3)$-Glc, $\beta$-D-Gal $p$ - $(1 \rightarrow$ 3)-Lac, and $\beta$-D-Galp-(1 $\rightarrow$ 4)-Lac. Interestingly, F417S shows the same relative composition of main GOS components (calculated as percentage mass of the total GOSs) throughout the conversion (Table 4). Separation and quantification by HPAEC-PAD of individual GOS produced during lactose conversion catalyzed by wild-type $H o B G L A$ and F417S are given in Fig. 3.

\section{Discussion}

When selecting the HoBGLA variants to be engineered, the designs (Fig. 1) were guided by work performed on homologous GH1 enzymes. Compared with HoBGLA N222F (Fig. 1b), the $T n \mathrm{Bgl1} \mathrm{A}$ variant $\mathrm{N} 220 \mathrm{~F}$ showed less dramatic effects on the kinetic parameters, i.e., $k_{\text {cat }}$ and $K_{\mathrm{m}}$ increased by 4 -fold and 2.8-fold, respectively, using $p$-nitrophenyl $\beta$-Dglucopyranoside ( $p \mathrm{NP}-\mathrm{Glc}$ ) relative to the wild type (Lundemo et al. 2013). There is no crystal structure available for $T n \mathrm{Bg} 11 \mathrm{~A}$, but molecular modeling suggests that the active site in $T n$ Bgll A is sufficiently different compared with that of $H o B G L A$ to account for the discrepancy. In addition, different substrates were used, which makes a direct comparison difficult. That aside, the impact of the mutation appears to have a more drastic effect on $\mathrm{HoBGLA}$.

For the $\mathrm{Tt} \beta$-gly $\mathrm{N} 282 \mathrm{~T}$ variant (corresponding to $\mathrm{Ho}$ BGLA N294T; Fig. 1c), $p$ NP-Glc was hydrolyzed with 4fold lower $k_{\text {cat }}$ and 27-fold higher $K_{\mathrm{m}}$ (Feng et al. 2005), while hydrolysis of $p$ NP-Gal was associated with a 8 -fold lower $k_{\text {cat }}$ and 6.5 -fold higher $K_{\mathrm{m}}$ value compared with the wild-type enzyme. The crystal structure of Tt $\beta$-gly N282T (PDB code 4BCE) did not reveal any significant structural changes that could explain the altered performance for this variant (Teze et al. 2014). The discrepancy in catalytic performance between the HoBGLA and $\mathrm{Tt} \beta$-gly variants is difficult to 
Table 3 Degree of lactose conversion, GOS yield, and individual GOS components

\begin{tabular}{lcccccc}
\hline Variants & WT & N222F & Y296F & F417S & N294T & F417Y \\
\hline Degree of lactose conversion (\%) & 99.2 & 49.9 & 70.4 & 79.5 & 39.3 & 97.4 \\
GOS yield (\% mass of total sugars) & 39.3 & 33.9 & 52.3 & 52.5 & 29.4 & 57.4 \\
GOS components $(\%$ mass of total GOS) & & & & & & \\
D-Galp- $(1 \rightarrow$ 3)-D-Gal & 8.2 & 0.0 & 0.0 & 0.0 & 0.0 & 2.8 \\
D-Galp-(1 $\rightarrow$ 6)-D-Gal & 7.0 & 2.6 & 0.0 & 0.0 & 0.0 & 4.7 \\
D-Galp-(1 $\rightarrow$ 3)-D-Glc & 8.8 & 3.7 & 10.5 & 25.1 & 22.8 & 13.6 \\
D-Galp-(1 $\rightarrow$ 6)-D-Glc & 16.7 & 24.8 & 0.0 & 0.0 & 0.0 & 8.8 \\
D-Galp-(1 $\rightarrow$ 3)-D-Lac & 5.1 & 10.9 & 16.9 & 6.2 & 31.0 & 13.4 \\
D-Galp-(1 $\rightarrow$ 4)-D-Lac & 2.9 & 0.0 & 1.4 & 2.0 & 3.3 & 3.3 \\
D-Galp-(1 $\rightarrow$ 6)-D-Lac & 45.4 & 57.9 & 66.3 & 66.5 & 42.6 & 53.1 \\
\hline
\end{tabular}

Degree of lactose conversion, GOS yield, and individual GOS components produced by the transgalactosylation reaction of wild-type $\mathrm{HoBGLA}$ and the variants using lactose as substrate after $8 \mathrm{~h}$ of reaction. The reactions were performed at $70{ }^{\circ} \mathrm{C}$ with an initial lactose concentration of $300 \mathrm{~g} / \mathrm{L}$ in sodium phosphate buffer $(\mathrm{pH} 6.0)$ and $1 \mathrm{mM} \mathrm{MgCl}_{2}$ using $0.35 \mathrm{mg}$ of purified enzyme rationalize from a structural viewpoint since the active sites are nearly identical, and the differences may be mainly accounted for by the different substrates used. Hydrolysis was severely compromised in the $\mathrm{Tt} \beta$-gly variant $\mathrm{Y} 284 \mathrm{~F}$ (Teze et al. 2014) and Agrobacterium $\beta$-glycosidase Abg Y298F (Gebler et al. 1995), which correspond to HoBGLA Y296F (Fig. 1d), and the tyrosine residue was assigned an important role to fine-tune the position of the nucleophile and to stabilize its deprotonated state (Gebler et al. 1995).

F417S (Fig. 1f) is the most catalytically impaired HoBGLA variant with respect to the hydrolytic reaction, but with lactose performing considerably better than cellobiose. Similarly, the corresponding $\mathrm{Tt} \beta$-gly variant $\mathrm{F} 401 \mathrm{~S}$ is also severely crippled catalytically, with no detectable hydrolytic activity on $p$-nitrophenyl $\beta$-D-glucoside ( $p$ NP-Glc) and $p$-nitrophenyl $\beta$-D-galactoside ( $p$ NP-Gal) (Feng et al. 2005). As for N282T, the crystal structure of $\mathrm{Tt} \beta$-gly F401S (PDB code $3 \mathrm{ZJK}$ ) offered no

Table 4 Time course of lactose conversion and formation of GOS by F417S

\begin{tabular}{lccccc}
\hline Time $(\mathrm{h})$ & 1 & 2 & 3 & 6 & 8 \\
\hline Degree of lactose conversion $(\%)$ & 30.2 & 46.2 & 55.8 & 73.3 & 79.5 \\
GOS yield (\% mass of total sugars) & 23.7 & 36.7 & 43.3 & 55.6 & 52.5 \\
GOS components (\% mass of total & GOS) & & & & \\
D-Galp-(1 $\rightarrow$ 3)-D-Glc & 18.8 & 23.1 & 24.7 & 25.5 & 25.1 \\
D-Galp-(1 $\rightarrow$ 4)-D-Lac & 2.7 & 2.6 & 2.8 & 3.0 & 2.0 \\
D-Galp-(1 $\rightarrow$ 3)-D-Lac & 7.4 & 6.9 & 6.5 & 6.1 & 6.2 \\
D-Galp-(1 $\rightarrow$ 6)-D-Lac & 70.9 & 67.3 & 65.8 & 65.3 & 66.5 \\
\end{tabular}

Time course of lactose conversion and formation of GOS during lactose conversion by the variant F417S. The reactions were performed at $70{ }^{\circ} \mathrm{C}$ at an initial lactose concentration of $300 \mathrm{~g} / \mathrm{L}$ in sodium phosphate buffer (pH 6.0) and $1 \mathrm{mM} \mathrm{MgCl}_{2}$ using $0.35 \mathrm{mg}$ of purified enzyme explanation for the loss of hydrolytic activity or improved $\mathrm{T} / \mathrm{H}$ ratio for the mutants (Teze et al. 2014), but the phenylalanine residue was suggested to be selectively important for transition state $\left(\mathrm{TS}^{*}\right)$ stabilization during the hydrolysis reaction, but of less significance for the transglycosylation reaction (Teze et al. 2014).

The rate of catalysis for cellobiose and lactose hydrolysis is only marginally affected in HoBGLA F417Y (Fig. 1g), and decreased $K_{\mathrm{m}}$ values are observed for both substrates. For the corresponding $P$. furiosus CelB variant $\mathrm{F} 426 \mathrm{Y}$, the turnover number decreased by approximately $30 \%$ while $K_{\mathrm{m}}$ was essentially unchanged using $p$ NP-Glc (Hansson et al. 2001). To improve the yield of transglycosylation products for $P$. furiosus CelB variant F426Y, the complementary replacement M424K was made, generating the variant M424K/F426Y (Hansson et al. 2001). As for F426Y, the double mutant displayed somewhat lower turnover number while $K_{\mathrm{m}}$ increased 1.5 -fold for $p$ NP-Glc compared with the wild type. The position in $\mathrm{Ho}$ BGLA equivalent to $\mathrm{M}^{424}$ in CelB is $\mathrm{K}^{415}$, and HoBGLA F417Y should therefore be compared with CelB M424K/ F426Y.

By dividing the specificity constant for cellobiose hydrolysis by that of lactose hydrolysis, a selectivity ratio [ $\left(k_{\text {catapp }} /\right.$ $\left.\left.K_{\mathrm{m}[\text { cellobiose }]}\right) /\left(k_{\text {cat,app }} / K_{\mathrm{m}[\text { lactose }]}\right)\right]$ is obtained, where a smaller ratio means improved performance with lactose over cellobiose as substrate. The selectivity ratio for the wild-type reaction with cellobiose measured at 50 and $70{ }^{\circ} \mathrm{C}$ shows that the preference for lactose is improved at higher temperatures, i.e., at $50{ }^{\circ} \mathrm{C}$, cellobiose is $\sim 9$ times favored over lactose, but at $70{ }^{\circ} \mathrm{C}$, cellobiose is only 2.8 times more favored over lactose. Thus, in a reaction where both cellobiose and lactose are present, elevated temperature improves lactose hydrolysis relative to cellobiose hydrolysis. Interestingly, $H o B G L A ~ F 417 S$ displays a change of substrate selectivity with lactose being 

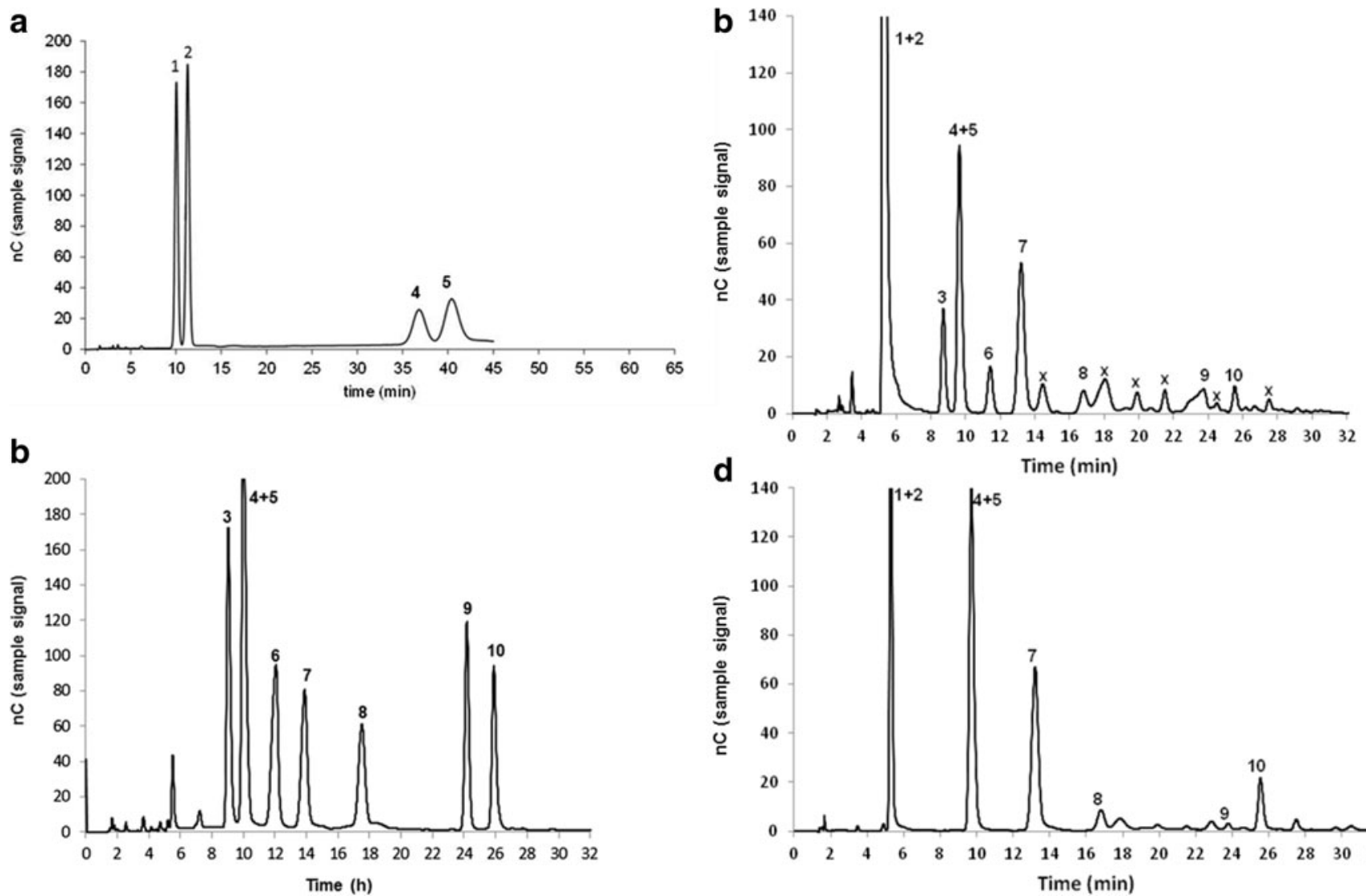

Fig. 3 Separation and quantification by HPAEC-PAD. Separation and quantification by HPAEC-PAD of authentic standards $(\mathbf{a}, \mathbf{b})$ and of the GOS mixtures produced during lactose conversion catalyzed by wildtype $H o$ BGLA (c) and HoBGLA F417S (d). The identified compounds are (1) galactose, (2) glucose, (3) D-Galp-( $1 \rightarrow$ 6)-D-Gal, (4) D-Galp- $(1 \rightarrow$ 6)-D-Glc (allolactose), (5) D-Galp-(1 $\rightarrow$ 4)-D-Glc (lactose), (6) D-Galp-

favored 11-fold over cellobiose (selectivity ratio 0.09 ). Improved selectivity ratios for lactose are also observed for the other variants, although cellobiose remains the preferred substrate.

In this study, the objective was to evaluate whether $\mathrm{Ho}$ BGLA can be engineered toward improved transgala ctosylation. With respect to transglycosylation performance, the variants $\mathrm{F} 417 \mathrm{~S}$ and $\mathrm{F} 417 \mathrm{Y}$ are of particular interest to address. The HoBGLA variant F417S (Table 3) produces mainly the trisaccharides $\beta$-D-Galp-(1 $\rightarrow 6)$-Lac and $\beta$-D-Gal $p$ - $(1 \rightarrow 3)$-Lac, and the disaccharides $\beta$-DGal $p-(1 \rightarrow 3)$-Gal and $\beta$-D-Gal $p-(1 \rightarrow 3)$-Glc, whereas for wild-type $H o B G L A$, several other disaccharides are also produced. The percentages of disaccharides versus trisaccharides were 33.2 versus $66.5 \%$ for $\mathrm{F} 417 \mathrm{Y}$, and 27.1 versus $72.7 \%$ for $\mathrm{F} 417 \mathrm{~S}$. The degree of lactose conversion of $H o B G L A$ F 417 Y is similar to that of the wild type, while the total GOSs has increased. The yield of total GOSs for F417Y is also close to that observed for some of the most efficient transglycosylating BGALs, e.g.,

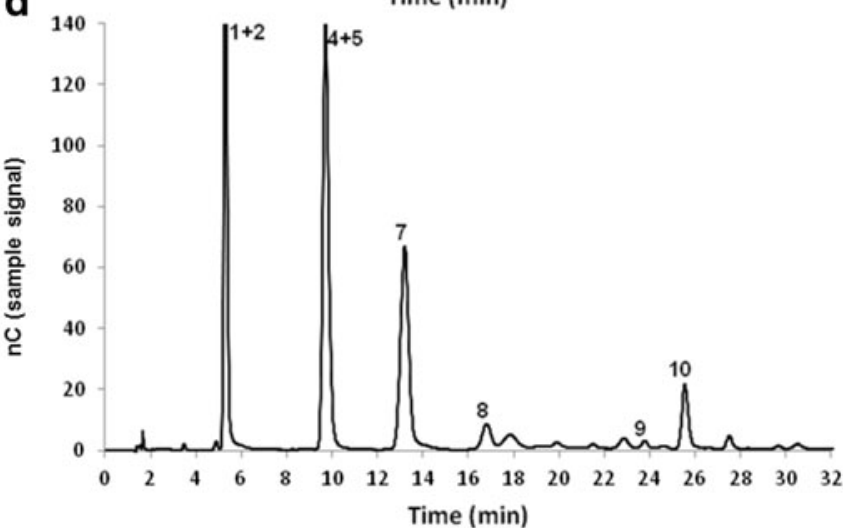

(1 $\rightarrow$ 3)-D-Gal, (7) D-Galp-(1 $\rightarrow$ 6)-Lac, (8) D-Galp-(1 $\rightarrow$ 3)-D-Glc, (9) D-Galp-( $1 \rightarrow 4)$-Lac, and $(10)$ D-Galp- $(1 \rightarrow 3)$-Lac. Products marked with an " $x$ " were not identified. Different conditions were used for HPAEC-PAD analysis to separate D-glucose-, D-galactose, lactose, and allolactose (gradient 1, panel (a)) as well as the other oligosaccharides (gradient 2, panels (b-d)). Details are given in the "Materials and methods" section

recombinant $\beta$-galactosidase from Bifidobacterium infantis for which $63 \%$ GOSs of the total sugars in the reaction mixture has been reported (Hung et al. 2001).

Several of the HoBGLA variants reported here show interesting properties for lactose conversion and GOS production. The variant F417S, as well as Y296F, produces $\beta$-D-Gal $p-(1 \rightarrow$ 6)-Lac in significantly higher amounts than the wild type and the other variants. Amino acid replacements in the -1 subsite, such as in F417S or Y296F, resulted in a significantly different spectrum of GOS components formed and could be of interest for tailoring GOS mixtures, e.g., for a higher content in trisaccharides. Variant $\mathrm{F} 417 \mathrm{Y}$ is almost as efficient as wild-type $H o$ BGLA in converting lactose but more competent for overall GOS production. This work shows that the thermostable BGLA from $H$. orenii can be successfully engineered toward higher GOS production and different GOS distributions depending on the requirements. Although the present enzyme variants would not contribute to industrial GOS production, they offer molecular insights into improved GOS yields by HoBGLA. 
Acknowledgments This work was facilitated by the Protein Science Facility at Karolinska Institutet/SciLifeLab (http://psf.ki.se). We thank Cindy Lorenz (BOKU, Vienna) for technical assistance. The authors have no conflict of interests to declare.

\section{Compliance with ethical standards}

Funding CD acknowledges grants from the Swedish Research Council Formas (Grant No. 2013-1741) and the Swedish Research Council VR (Grant No. 2013-5717). BG, RK, and THN acknowledge the support from the Austrian Science Fund (FWF Projects P25313-B20, P24868-B22). BG also thanks for the support from doctoral program Biomolecular Technology of Proteins (BioToP; grant FWF-W1224) of the Austrian Science Fund (FWF).

Conflict of interest The authors declare that there are no conflict of interest.

Ethical approval This article does not contain any studies with human participants or animals performed by any of the authors.

Open Access This article is distributed under the terms of the Creative Commons Attribution 4.0 International License (http:// creativecommons.org/licenses/by/4.0/), which permits unrestricted use, distribution, and reproduction in any medium, provided you give appropriate credit to the original author(s) and the source, provide a link to the Creative Commons license, and indicate if changes were made.

\section{References}

Arreola SL, Intanon M, Suljic J, Kittl R, Pham NH, Kosma P, Haltrich D, Nguyen TH (2014) Two $\beta$-galactosidases from the human isolate Bifidobacterium breve DSM 20213: molecular cloning and expression, biochemical characterization and synthesis of galacto-oligosaccharides. PLoS One 9:e104056

Cayol I, Ollivier B, Patel BKC, Prensier G, Guezennec J, Garcia JL (1994) Isolation and characterization of Halothermothrix orenii gen. nov., sp. nov., a halophilic, thermophilic, fermentative, strictly anaerobic bacterium. Int J Syst Bacteriol 44:534-540

Chockchaisawasdee S, Athanasopoulos VI, Niranjan K, Rastall RA (2005) Synthesis of galacto-oligosaccharide from lactose using $\beta$ galactosidase from Kluyveromyces lactis: studies on batch and continuous UF membrane-fitted bioreactors. Biotechnol Bioeng 89: 434-443

Feng HY, Drone J, Hoffmann L, Tran V, Tellier C, Rabiller C, Dion M (2005) Converting a $\beta$-glycosidase into a $\beta$-transglycosidase by directed evolution. J Biol Chem 280:37088-37097

Gebler JC, Trimbur DE, Warren RAJ, Aebersold R, Namchuk M, Withers SG (1995) Substrate-induced inactivation of a crippled $\beta$ glucosidase mutant: identifcation of the labeled amino acid and mutagenic analysis of its role. Biochemistry 34:14547-14553

Gosling A, Stevens GW, Barber AR, Kentish SE, Gras SL (2010) Recent advances refining galactooligosaccharide production from lactose. Food Chem 121:307-318

Goulas T, Goulas A, Tzortzis G, Gibson GR (2009) Expression of four $\beta$ galactosidases from Bifidobacterium bifidum NCIMB41171 and their contribution on the hydrolysis and synthesis of galactooligosaccharides. Appl Microbiol Biotechnol 84:899-907

Hansson T, Kaper T, van der Oost J, de Vos WM, Adlercreutz P (2001) Improved oligosaccharide synthesis by protein engineering of $\beta$ glucosidase CelB from hyperthermophilic Pyrococcus furiosus. Biotech Bioeng 73:203-210
Hassan N, Nguyen TH, Intanon M, Kori LD, Patel BKC, Haltrich D, Divne C, Tan TC (2015) Biochemical and structural characterization of a thermostable $\beta$-glucosidase from Halothermothrix orenii for galacto-oligosaccharide synthesis. Appl Microbiol Biotechnol 99: $1731-1744$

Hung MN, Xia Z, Hu NT, Lee BH (2001) Molecular and biochemical analysis of two $\beta$-galactosidases from Bifidobacterium infantis HL96. Appl Microbiol Biotechnol 67:4256-4263

Hung MN, Lee BH (2002) Purification and characterization of a recombinant $\beta$-galactosidase with transgalactosylation activity from Bifidobacterium infantis HL96. Appl Microbiol Biotechnol 58: 439-445

Iqbal S, Nguyen TH, Nguyen HA, Nguyen TT, Maischberger T, Kittl R, Haltrich D (2011) Characterization of a heterodimeric GH2 $\beta$ galactosidase from Lactobacillus sakei Lb790 and formation of prebiotic galacto-oligosaccharides. J Agric Food Chem 59:3803-3811

Jørgensen F, Hansen OC, Stougaard P (2001) High-efficiency synthesis of oligosaccharides with a truncated $\beta$-galactosidase from Bifidobacterium bifidum. Appl Microbiol Biotechnol 57:647-652

Kori L, Hofmann A, Patel BKC (2011) Expression, purification and preliminary crystallographic analysis of the recombinant $\beta$ glucosidase (BglA) from the halothermophile Halothermothrix orenii. Acta Crystallogr F Struct Biol Commun 67:111-113

Kunst A, Draeger B, Ziegernhorn J (1988) Colorimetric methods with glucose oxidase and peroxidase. In: Methods of Enzymatic Analysis (Bergmeyer HU, Bergmeyer J, Grassl M, editors), pp. 178-185. VCH Publishers 1988, third edition, Weinheim, Germany.

Liu GX, Kong J, Lu WW, Kong WT, Tian H, Tian XY, Huo GC (2011) ßgalactosidase with transgalactosylation activity from Lactobacillus fermentum K4. J Dairy Sci 94:5811-5820

Lombard V, Golaconda RH, Drula E, Coutinho PM, Henrissat B (2014) The carbohydrate-active enzymes database (CAZy) in 2013. Nucleic Acids Res 42:D490-D495

Lundemo P, Adlercreutz P, Nordberg Karlsson E (2013) Improved transferase/hydrolase ratio through rational design of a family $1 \beta$ glucosidase from Thermotoga neapolitana. Appl Environ Microbiol 79:3400-3405

Maischberger T, Nguyen TH, Sukyai P, Kittl R, Riva S, Ludwig R, Haltrich D (2008) Production of lactose-free galacto-oligosaccharide mixtures: comparison of two cellobiose dehydrogenases for the selective oxidation of lactose to lactobionic acid. Carbohydr Res 343:2140-2147

Maischberger T, Leitner E, Nitisinprasert S, Juajun O, Yamabhai M, Nguyen TH, Haltrich D (2010) $\beta$-galactosidase from Lactobacillus pentosus: purification, characterization and formation of galacto-oligosaccharides. Biotechnol J 5:838-847

Mavromatis K, Ivanova N, Anderson I, Lykidis A, Hooper SD, Sun H, Kunin V, Hugenholtz P, Patel B, Kyrpides NC (2009) Genome analysis of the anaerobic thermohalophilic bacterium Halothermothrix orenii. PLoS One 4:e4192

Mijts B, Patel BKC (2001) Random sequence analysis of genomic DNA of an anaerobic, thermophilic, halophilic bacterium, Halothermothrix orenii. Extremophiles 5:61-69

Nakkharat P, Haltrich D (2006) Purification and characterisation of an intracellular enzyme with $\beta$-glucosidase and $\beta$-galactosidase activity from the thermophilic fungus Talaromyces thermophilus CBS 236.58. J Biotechnol 123:304-313

Neri DFM, Balcao VM, Costa RS, Rocha ICAP, Ferreira EMFC, Torres DPM, Rodrigues LRM, Carvalho Jr LB, Teixeira JA (2009) Galacto-oligosaccharides production during lactose hydrolysis by free Aspergillus oryzae $\beta$-galactosidase and immobilized on magnetic polysiloxane-polyvinyl alcohol. Food Chem 115:92-99

Nguyen TH, Splechtna B, Steinböck M, Kneifel W, Lettner HP, Kulbe KD, Haltrich D (2006) Purification and characterization of two novel $\beta$-galactosidases from Lactobacillus reuteri. J Agric Food Chem 54:4989-4998 
Nguyen TT, Nguyen HA, Arreola SL, Mlynek G, Djinovic-Carugo K, Mathiesen G, Nguyen TH, Haltrich D (2012) Homodimeric $\beta$ galactosidase from Lactobacillus delbrueckii subsp. Bulgaricus DSM 20081: expression in Lactobacillus plantarum and biochemical characterization. J Agric Food Chem 60:1713-1721

Osman A, Tzortzis G, Rastall RA, Charalampopoulos D (2012) BbgIV is an important Bifidobacterium $\beta$-galactosidase for the synthesis of prebiotic galactooligosaccharides at high temperatures. J Agric Food Chem 60:740-748

Petzelbauer I, Reiter A, Splechtna B, Kosma P, Nidetzky B (2000) Transgalactosylation by thermostable $\beta$-glycosidases from Pyrococcus furiosus and Sulfolobus solfataricus. Eur J Biochem 267:5055-5066

Rodriguez-Colinas B, de Abreu MA, Fernandez-Arrojo L, de Beer R, Poveda A, Jiminez-Barbero J, Haltrich D, Ballesteros Olmo AO, Fernandez-Lobato M, Plou FJ (2011) Production of galactooligosaccharides by the $\beta$-galactosidase from Kluyveromyces lactis: comparative analysis of permeabilized cells versus soluble enzyme. J Agric Food Chem 59:10477-10484

Rodriguez-Colinas B, Poveda A, Jimenez-Barbero J, Ballesteros AO, Plou FJ (2012) Galacto-oligosaccharide synthesis from lactose solution or skim milk using the $\beta$-galactosidase from Bacillus circulans. J Agric Food Chem 60:6391-6398

Rodriguez-Colinas B, Fernandez-Arrojo L, Ballesteros AO, Plou FJ (2013) Galactooligosaccharides formation during enzymatic hydrolysis of lactose: towards a prebiotic-enriched milk. Food Chem 145: 388-394

Savitsky P, Bray J, Cooper CDO, Marsden BD, Mahajan P, BurgessBrown NA, Gileadi O (2010) High-throughput production of human proteins for crystallization: the SGC experience. J Struct Biol 172:3-13

Smithers GW (2008) Whey and whey proteins-from 'gutter-to-gold'. Int Dairy J 18:695-704

Splechtna B, Nguyen TH, Haltrich D (2007) Comparison between discontinuous and continuous lactose conversion processes for the production of prebiotic galacto-oligosaccharides using $\beta$-galactosidase from Lactobacillus reuteri. J Agric Food Chem 55:6772-6777

Teze D, Hendrickx J, Czjzek M, Ropartz D, Sanejouand YH, Tran V, Tellier C, Dion M (2014) Semi-rational approach for coverting a GH1 $\beta$-glucosidase into a $\beta$-transglucosidase. Prot Eng Des Sel 27:13-19

Torres DPM, Gonçalves MPF, Teixeira JA, Rodrigues LR (2010) Galacto-oligosaccharides: production, properties, applications, and significance as prebiotics. Comprehensive Rev Food Sci Food Safety 9:438-454

Urrutia P, Rodriguez-Colinas B, Fernandez-Arrojo L, Ballesteros AO, Wilson L, Illanes A, Plou FJ (2013) Detailed analysis of galactooligosaccharides synthesis with $\beta$-galactosidase from Aspergillus oryzae. J Agric Food Chem 61:1081-1087

Wu Y, Yuan S, Chen S, Wu D, Chen J, Wu J (2013) Enhancing the production of galacto-oligosaccharides by mutagenesis of Sulfolobus solfataricus $\beta$-galactosidase. Food Chem 138: $1588-1595$

Yu L, Sullivan DJO (2013) Production of galactooligosaccharides using a hyperthermophilic $\beta$-galactosidase in permeabilized cells of Lactococcus lactis. J Dairy Sci 97:694-703 